\title{
Discrete-Log With Compressible Exponents
}

\author{
Yacov Yacobi \\ Bellcore \\ 445 South St., Morristown, NJ 07960
}

\begin{abstract}
Many key distribution systems are based on the assumption that the DiscreteLog (DL) problem is hard. The implementations could be more efficient if a significantly smaller exponent could be used, without lowering the complexity of the DL problem. When the exponent is known to reside in interval of size $w$, the DL problem can be computed in time $O(\sqrt{w})$, using Pollard's "Lambda method for catching Kangaroos".

Suppose we want a level of security of 300 years on a 1 MIP machine, with $1 \mathrm{~K}$ bit operations per instruction. Then $w=2^{127}$ currently seems sufficient (with 512 bit modulus). It is not clear, however, whether methods other than "Kangaroo" exist, with lower complexity.

Let $s$ and $m$ denote the number of squarings and multiplications, respectively, required to exponentiate. It is well known that $s$ roughly equals the size in bits of the exponent $(L)$, and $m$ is roughly $1.5 \cdot L / \lg _{2}(L)$, for the most efficient methods, in the practical range.

We show that by using an exponent which is known to be compressible by a factor $\eta$, using the Ziv-Lempel method, we reduce $m$ exponentially in $\eta$, on the average (integer multiplications may be more than twice as expensive as squarings, hence this is not negligible).

This can be used to speed up cryptographic key distribution systems of the Diffie-Hellman family. However, it is not clear how safe compressible exponents are.
\end{abstract}

\section{Introduction}

Many key distribution systems are based on the assumption that the Discrete-Log (DL) problem is hard. The implementations could be more efficient if a significantly smaller exponent could be used, without lowering the complexity of the DL problem. When the exponent is known to reside in interval of size $w$, the DL problem can be computed in time $O(\sqrt{w})$, using Pollard's "Lambda method for catching Kangaroos" $[\mathrm{P}]$. This is a randomized algorithm, with controllable error probability $\epsilon>0$, which 
tree, where the path $e_{i}$ from the root to node $i$ is a segment of the exponent, and node $i$ contains the partial result $x^{e_{i}}$. The structure of the tree makes it easy to add a new leaf, given its parent. We describe first a subroutine that creates the binary tree, and then the main program, which uses the tree to compute $x^{n}$.

The exponent is called here seq, and is treated sometimes as an integer, and sometimes as a sequence of bits. Each segment $e_{i}$ of the exponent is an odd number of length $L_{i} \geq 1$, and is preceded (to the right) by $Z_{i} \geq 0$ zeroes. subroutine build-tree

begin

- Init: Store $e_{0}=1 ; Z_{0} \leftarrow L_{-1} \leftarrow 0$ in node 0 , and set $L_{0} \leftarrow 0$. Put parse 0 right of seq; $i \leftarrow 1$;

- While there are more symbols in seq do

- begin

$-L_{i} \leftarrow 0 ; \quad Z_{i} \leftarrow 0$.

- Scan seq from the $i-1_{t h}$ parse to the left.

- While the new symbol (ns) $=0 Z_{i} \leftarrow Z_{i}+1$.

- (ns=1) $L_{i} \leftarrow L_{i}+1$; start following the path of the tree defined by $s e q$, incrementing $L_{i}$, until a leaf is reached.

- Scan one more symbol ( $n s$ ), add a new arc from the last visited leaf, label it $n s$, add a new leaf and a new parse, and label them $i . e_{i}$ is the integer represented by the segment which starts at parse $i-1$ and ends at parse $i$, not including leading zeroes to the right.

- Compute $x^{e_{i}}$, and store it together with $L_{i-1}=\left|e_{i-1}\right|, e_{i-1}$, and $Z_{i}$ in leaf $i$.

$-i \leftarrow i+1$;

- end;

end.

Let $k=\max (i)$. The exponent looks as follows. $n=e_{k} 0^{z_{k}} e_{k-1} 0^{z_{k-1}} \ldots e_{1} 0^{z_{1}}$ 


\section{Algorithm 1:}

begin

- Call build-tree subroutine.

- $x^{n} \leftarrow\left(\left(\ldots\left(\left(x^{e_{4}}\right)^{2^{x_{k}+L_{k-1}}} \cdot x^{e_{k-1}}\right)^{2^{x_{k-1}+L_{k-1}}} \cdot x^{e_{k-2}} \ldots\right)^{2^{x_{2}+L_{1}}} \cdot x^{e_{1}}\right)^{2^{x_{1}}}$

end.

\section{Remarks :}

- $e_{i-1}$ is stored in "node $e_{i}$ " as a back pointer, later used by algorithm 1 to determine the right sequencing of the partial results.

- The method is general, and applies to any multiplicative group.

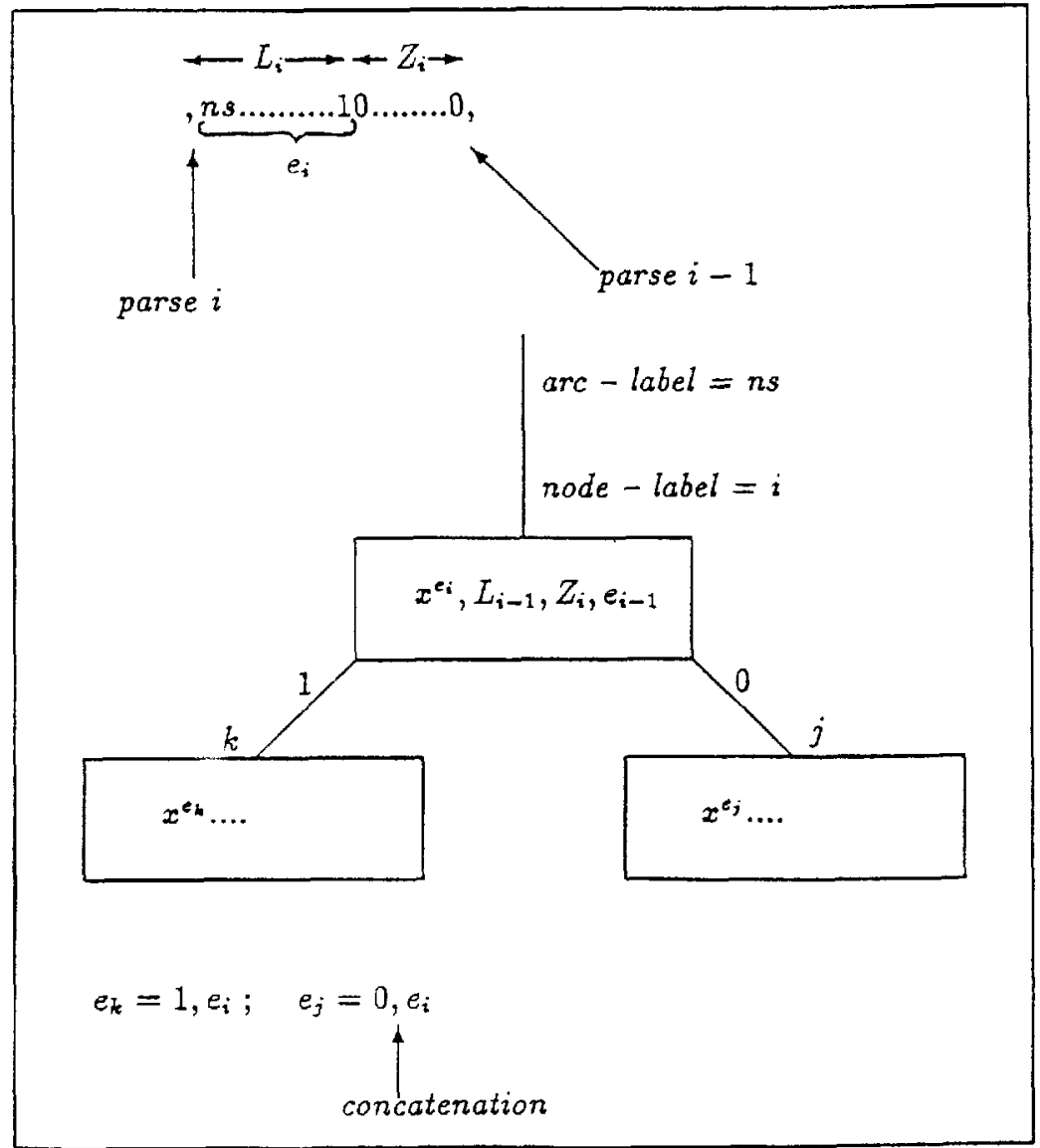

The general structure of the tree 
The following theorem and its proof appear in [Y]. As before, let $L=\log (n)$ the length of the exponent.

Theorem: The average time complexity of Algorithm 1 is $l(n)=L-(\log (L)-\log \log (L)) / 2+1.5 \cdot(L / \log (L)+o(L / \log (L))$.

\section{Compressible exponents}

Roughly speaking, if a sequence is ZL-compressible then its compression tree is not balanced, and on the average, node-names are shorter than the corresponding exponent segments, i.e. the tree has relatively few nodes. Let $k$ be the number of nodes of a noncompressible sequence, and $k^{\prime}$ the number of nodes of another sequence of the same length, which is compressible by a factor $\eta<1$. Let $N$ denote the expected length of a node-name (similarly $N^{\prime}$ for the compressible case). Then roughly $k=2^{N}$, and since $N^{\prime}=\eta \cdot N$, we get $k^{\prime}=2^{N^{\prime}}=k^{\eta}$. The number of multiplications in the compressible case is $m^{\prime}=c \cdot k^{\prime}, 1<c<2$ compared with $m=1.5 \cdot k$, i.e. the saving is asymptotically exponential in $\eta$.

If the tree is skewed to the right (fewer ones), then $c<1.5$, while if the tree is skewed to the left, $c>1.5$.

Some implementors use exponents of low Hamming weight; thus gaining in the linear factor $(c<1.5)$, this may be combined with the new algorithm to gain twice: use low weight exponents $(c<1.5)$, which are also ZL-compressible $(\eta<1)$.

\section{References}

[L ] A.K. Lenstra, and H.W. Lenstra, Jr.: "Algorithms in Number Theory," Technical Report 87-008, Univ. of Chicago, Dept. of CS, May 1987

[P ] J.M. Pollard, "Monte Carlo Methods for Index Computation $(\bmod p)$," Math. Comp. 32 (1978), 918-924

[Y] Y. Yacobi, "Exponentiating faster with addition chains," Proceedings of Eurocrypt'90.

[ZL ] J. Ziv, and A. Lempel: "Compression of individual sequences via variable rate coding," IEEE Trans. Inf. Th. Vol. IT-24, No. 5, Sep. 1978 